\title{
SpS1-Dust and gas clearing in transitional disks
}

\author{
Joanna M. Brown ${ }^{1}$ \\ ${ }^{1}$ Max-Planck-Institut für extraterrestrische Physik, Garching bei München, Germany
}

Understanding how disks dissipate is essential to studies of planet formation. Infrared observations of young stars demonstrate that optically-thick circumstellar disks disappear from around half the stars in low-mass star-forming regions by an age of $3 \mathrm{Myr}$ and are almost entirely absent in $10 \mathrm{Myr}$ old associations (e.g. Haisch et al., 2001). Accretion ceases on the same approximate timescale (e.g. Calvet et al. 2005). The disappearence of gas and dust - planetary building material - places stringent limits on the timescales of giant planet formation. During this crucial interval, planet(esimal)s form and the remaining disk material is accreted or dispersed. Mid-infrared spectrophotometry of protoplanetary disks has revealed a small sub-class of objects in the midst of losing their disk material. These disks have spectral energy distributions (SEDs) suggestive of large inner gaps with low dust content, often interpreted as a signature of young planets. Such objects are still rare although Spitzer surveys have significantly increased the number of known transitional objects (e.g. Brown et al. 2007, D'Alessio et al., 2005). However, spectrophotometric signatures are indirect and notoriously difficult to interpret as multiple physical scenarios can result in the same SED. Recent direct imaging from millimeter interferometry has confirmed the presence of large inner holes in transitional disks, providing additional constraints and lending confidence to current SED interpretations (Brown et al. 2008, Brown et al. 2009, Andrews et al. 2009, Isella et al., 2009).

Most transitional disks are discovered through the properties of their dust emission and much less is known about the gas content. However, the state of the gas has strong implications for the method of disk evolution. Photoevaporation requires a complete absence of gas in the inner regions, while grain growth and settling can occur with high gas densities. Stellar and planetary companions may allow gas in the inner regions depending on the mass and orbital radius of the companion. Spectroscopy provides tracers of the gas throughout the disk and reveals both the physical and chemical structure. For example, high resolution 5 micron spectra reveal that CO gas is often present inside the dust holes Salyk et al., 2009, Pontoppidan et al., 2008. Prospects for the future are particularly good as Herschel, ALMA and the next generation of ground-based high resolution spectrometers will provide additional clarity on current results as well as access to a larger sample of transitional disks too faint for current instruments.

\section{References}

Andrews, S. M., Wilner, D. J., Hughes, A. M., Qi, C., \& Dullemond, C. P., 2009, ApJ, 700, 1502 Brown, J. M. et al., 2007, ApJL, 664, L107

Brown, J. M., Blake, G. A., Qi, C., Dullemond, C. P., \& Wilner, D. J., 2008, ApJL, 675, L109

Brown, J. M., Blake, G. A., Qi, C., Dullemond, C. P., Wilner, D. J., \& Williams, J. P., 2009, ApJ, 704, 496

Calvet, N. et al., 2005, AJ, 129, 935

D'Alessio, P. et al. 2005, ApJ, 621, 461

Haisch, Jr., K. E., Lada, E. A., \& Lada, C. J., 2001, ApJL, 553, L153

Isella, A., Carpenter, J. M., \& Sargent, A. I., 2009, ApJ, 701, 260

Pontoppidan, K. M., Blake, G. A., van Dishoeck, E. F., Smette, A., Ireland, M. J., \& Brown, J., 2008, ApJ, 684, 1323

Salyk, C., Blake, G. A., Boogert, A. C. A., \& Brown, J. M., 2009, ApJ, 699, 330 